\title{
Generating functions for generalized mock theta functions
}

\author{
Sameena Saba \\ Lucknow University, Lucknow, India
}

Email address:

saba080284@gmail.com (S. Saba)

\section{To cite this article:}

Sameena Saba. Generating Functions for Generalized Mock Theta Functions, Pure and Applied Mathematics Journal. Vol. 2, No. 2, 2013, pp. 62-70. doi: 10.11648/j.pamj.20130202.13

Abstract: We consider generalized mock theta functions and give generating functions for the partial generalized mock theta functions.

Keywords: Generating Function, Mock Theta Function And Hypergeometric Series

\section{Introduction}

Mock theta functions have been generalized in [6] [7] [9]. We give below the definitions for the generalized third order, fifth order, sixth order, eighth order and tenth order mock theta functions.

This paper aims at presenting generating functions for these generalized functions. As a tool we have used simple expansion formula of Srivastava [8]. The generating functions can be suitably applied to yield numerous further results involving these generalized functions.

A great surge of activities in the theory of q-series has been witnessed in recent years with this objective in mind we give generating functions for a very important class of special functions- mock theta functions.

\section{Definitions and Notations}

A generalized basic hypergeometric function with base $q$ is defined as:

$$
\begin{gathered}
r \phi_{s}\left[\begin{array}{l}
a_{1}, a_{2} \cdots, a_{r} \\
b_{1}, b_{2} \cdots, b_{s} ; q, z
\end{array}\right] \\
=\sum_{n=0}^{\infty} \frac{\left(a_{1} ; q\right)_{n} \cdots\left(a_{r} ; q\right)_{n}}{\left(b_{1} ; q\right)_{n} \cdots\left(b_{s} ; q\right)_{n}(q ; q)_{n}}\left[(-1)^{n} q^{\frac{n^{2}-n}{2}}\right]^{1+s-r} z^{n}
\end{gathered}
$$

For $r=s+1$, the above series is convergent for $|z|<1$, for $r \leq s$, the above series is convergent for all $z$ and for $r>s+1$, the series diverges for all $z$ except $z=0$.

Also,

$$
\begin{gathered}
r \phi_{s}\left[\begin{array}{l}
a_{1}, a_{2} \cdots, a_{r} \\
b_{1}, b_{2} \cdots, b_{s} ; q, z
\end{array}\right]_{m} \\
=\sum_{n=0}^{m} \frac{\left(a_{1} ; q\right)_{n} \cdots\left(a_{r} ; q\right)_{n}}{\left(b_{1} ; q\right)_{n} \cdots\left(b_{s} ; q\right)_{n}(q ; q)_{n}}\left[(-1)^{n} q^{\frac{n^{2}-n}{2}}\right]^{1+s-r} z^{n}
\end{gathered}
$$

denotes the partial sum of the generalized basic hypergeometric series.

Here we have used the standard q-shifted factorials defined as

$$
\begin{gathered}
(a)_{n}=(a ; q)_{n}=\prod_{k=1}^{n}\left(1-a q^{k-1}\right) \\
(a)_{\infty}=(a ; q)_{\infty}=\prod_{k=1}^{\infty}\left(1-a q^{k-1}\right), \quad|q|<1 . \\
(a)_{0}=1 .
\end{gathered}
$$

Main result

We shall be using the following identity in getting the generating functions for generalized mock theta functions.

$$
\sum_{m=0}^{\infty} t^{m} \sum_{r=0}^{m} \alpha_{r}=\left[\sum_{r=0}^{\infty} \alpha_{r} t^{r}\right] \sum_{n=0}^{\infty} t^{n}, \quad|t|<1
$$

This identity is easily deduced from the identity of Srivastava [8]:

$$
\sum_{r=0}^{p} \alpha_{r} \beta_{r}=\beta_{p+1} \sum_{r=0}^{p} \alpha_{r}+\sum_{m=0}^{p}\left(\beta_{m}-\beta_{m+1}\right) \sum_{r=0}^{m} \alpha_{r}
$$


Take $\beta_{r}=t^{r},|t|<1$.

$$
\begin{aligned}
& \sum_{r=0}^{p} \alpha_{r} t^{r}=t^{p+1} \sum_{r=0}^{p} \alpha_{r}+(1-t) \sum_{m=0}^{p} t^{m} \sum_{r=0}^{m} \alpha_{r} \\
& \text { Let } p \rightarrow \infty, \\
& \sum_{m=0}^{\infty} t^{m} \sum_{r=0}^{m} \alpha_{r}=\frac{1}{1-t} \sum_{r=0}^{\infty} \alpha_{r} t^{r}=\sum_{r=0}^{\infty} \alpha_{r} t^{r} \sum_{n=0}^{\infty} t^{n}
\end{aligned}
$$

\section{Definition of Generalized Functions}

To distinguish third order mock theta functions from tenth order mock theta functions we put suffix $R$ in the symbol for third order mock theta functions.

The generalized third order mock theta functions [9]:

$$
\begin{gathered}
f_{R}(t, \alpha, \beta, z ; q)=\frac{1}{(t)_{\infty}} \sum_{n=0}^{\infty} \frac{(t)_{n} q^{n^{2}-4 n+n \beta} \alpha^{n} z^{2 n}}{(-z ; q)_{n}(-\alpha z / q ; q)_{n}} \\
\phi_{R}(t, \alpha, \beta, z ; q)=\frac{1}{(t)_{\infty}} \sum_{n=0}^{\infty} \frac{(t)_{n} q^{n^{2}-3 n+n \beta} z^{2 n}}{\left(-\alpha z^{2} / q ; q^{2}\right)_{n}} \\
\psi_{R}(t, \alpha, \beta, z ; q)=\frac{1}{(t)_{\infty}} \sum_{n=0}^{\infty} \frac{(t)_{n} q^{n^{2}-n+n \beta} z^{2 n+1}}{\left(\alpha z^{2} / q^{2} ; q^{2}\right)_{n+1}}, \\
v_{R}(t, \alpha, \beta, z ; q)=\frac{1}{(t)_{\infty}} \sum_{n=0}^{\infty} \frac{(t)_{n} q^{n^{2}-2 n+n \beta} z^{2 n}}{\left(-\alpha^{2} z^{2} / q^{3} ; q^{2}\right)_{n+1}} \\
\omega_{R}(t, \alpha, \beta, z ; q)=\frac{1}{(t)_{\infty}} \sum_{n=0}^{\infty} \frac{(t)_{n} q^{2 n^{2}-5 n-4+n \beta} \alpha^{2 n_{z}} z^{4}(n+1)}{\left(z^{2} / q ; q^{2}\right)_{n+1}\left(\alpha^{2} z^{2} / q^{3} ; q^{2}\right)_{n+1}} \\
\chi_{R}(t, \beta, z ; q)=\frac{1}{(t)_{\infty}} \sum_{n=0}^{\infty} \frac{(t)_{n} q^{n^{2}-3 n+n \beta} z^{2 n}}{(v z ; q)_{n}\left(-v^{2} z ; q\right)_{n}}
\end{gathered}
$$

and

$$
\begin{gathered}
\rho_{R}(t, \beta, z ; q)= \\
\frac{z^{4}}{q^{4}} \frac{1}{(t)_{\infty}} \sum_{n=0}^{\infty} \frac{(t)_{n} q^{2 n^{2}-3 n+n \beta} z^{4 n}}{\left(v^{2} z^{2} / q ; q^{2}\right)_{n+1}\left(v^{-2} z^{2} / q ; q^{2}\right)_{n+1}}
\end{gathered}
$$

where $v=e^{\frac{\pi i}{3}}$.

For $\beta=1$ and $z=q$ we have generalized five third order mock theta functions namely $f, \phi, \psi, v, \omega$ of Andrews [2]. For $t=0, \beta=1, \alpha=q$ and $z=q$ the generalized functions $f, \phi, \psi$ and $\chi$ reduce to the third order mock theta functions of Ramanujan and $\omega, v$ and $\rho$ to the third order mock theta functions of Watson [10].

The generalized fifth order mock theta functions [6]:

$$
\begin{aligned}
& f_{0}(t, \alpha, z ; q)=\frac{1}{(t)_{\infty}} \sum_{n=0}^{\infty} \frac{(t)_{n} q^{n^{2}-3 n+n \alpha} z^{2 n}}{(-z ; q)_{n}}, \\
& \phi_{0}(t, \alpha, z ; q) \\
& =\frac{1}{(t)_{\infty}} \sum_{n=0}^{\infty} \frac{(t)_{n} q^{n^{2}+n+n \alpha}\left(-q^{3} / z^{2} ; q^{2}\right)_{n}}{z^{2 n}},
\end{aligned}
$$

$$
\begin{aligned}
& \psi_{0}(t, \alpha, z ; q) \\
& =\frac{1}{(t)_{\infty}} \sum_{n=1}^{\infty} \frac{(t)_{n} q^{n(n+1) / 2+n \alpha}\left(-q^{2} / z ; q\right)_{n-1}}{z^{n}}, \\
& F_{0}(t, \alpha, z ; q)=\frac{1}{(t)_{\infty}} \sum_{n=0}^{\infty} \frac{(t)_{n} q^{2 n^{2}-5 n+n \alpha} z^{4 n}}{\left(z^{2} / q ; q^{2}\right)_{n}} \\
& f_{1}(t, \alpha, z ; q)=\frac{1}{(t)_{\infty}} \sum_{n=0}^{\infty} \frac{(t)_{n} q^{n^{2}-2 n+n \alpha} z^{2 n}}{(-z ; q)_{n}} \\
& \phi_{1}(t, \alpha, z ; q) \\
& =\frac{q^{5}}{z^{4}} \frac{1}{(t)_{\infty}} \sum_{n=0}^{\infty} \frac{(t)_{n} q^{n^{2}+3 n+n \alpha}\left(-q^{3} / z^{2} ; q^{2}\right)_{n}}{z^{2 n}}, \\
& \psi_{1}(t, \alpha, z ; q) \\
& =\frac{q}{z} \frac{1}{(t)_{\infty}} \sum_{n=0}^{\infty} \frac{(t)_{n} q^{n(n+1) / 2+n \alpha}\left(-q^{2} / z ; q\right)_{n}}{z^{n}}, \\
& \chi_{0}(t, \alpha, z ; q)=\frac{1}{(t)_{\infty}} \sum_{n=0}^{\infty} \frac{(t)_{n} q^{n \alpha}(z ; q)_{n}}{\left(z^{2} q^{-1} ; q\right)_{2 n}} \\
& F_{1}(t, z ; q)=\frac{1}{(t)_{\infty}} \sum_{n=0}^{\infty} \frac{(t)_{n} q^{2 n^{2}-3 n+n \alpha} z^{4 n}}{\left(z^{2} / q ; q^{2}\right)_{n+1}},
\end{aligned}
$$

And

$\chi_{1}(t, \alpha, z ; q)=\frac{1}{(t)_{\infty}} \sum_{n=0}^{\infty} \frac{(t)_{n} q^{n \alpha}(z ; q)_{n}}{\left(z^{2} q^{-1} ; q\right)_{2 n+1}}$.

For $t=0, \alpha=1$ and $z=q$ these generalized functions reduce to Ramanujan's mock theta functions of order five.

The generalized sixth order mock theta functions [9]:

$$
\begin{aligned}
& =\frac{1}{(t)_{\infty}} \sum_{n=0}^{\infty} \frac{(-1)^{n}(t)_{n} q^{n(n-3)+n \alpha} z^{2 n}\left(z^{2} / q ; q^{2}\right)_{n}}{\left(-z^{2} / q ; q\right)_{2 n}},(1) \\
& =\frac{1}{(t)_{\infty}} \sum_{n=0}^{\infty} \frac{(-1)^{n}(t)_{n} q^{n(n-1)+n \alpha} z^{2 n+1}\left(z^{2} / q ; q^{2}\right)_{n}}{\left(-z^{2} / q ; q\right)_{2 n+1}} \\
& =\frac{1}{(t)_{\infty}} \sum_{n=0}^{\infty} \frac{(t)_{n} q^{n(n-3) / 2+n \alpha} z^{n}(-z ; q)_{n}}{\left(z^{2} / q ; q^{2}\right)_{n+1}} \\
& =\frac{1}{2(t)_{\infty}} \sum_{n=0}^{\infty} \frac{(t)_{n} q^{n(n-1) / 2+n \alpha} z^{n+1}(-z / q ; q)_{n+1}}{\left(z^{2} / q ; q^{2}\right)_{n+1}} \\
& \lambda(t, \alpha, z ; q) \\
& =\frac{1}{(t)_{\infty}} \sum_{n=0}^{\infty} \frac{(t)_{n}(-1)^{n} q^{n \alpha}\left(q^{3} / z^{2} ; q^{2}\right)_{n}}{\left(-q^{2} / z ; q\right)_{n}}
\end{aligned}
$$




$$
\begin{gathered}
=\frac{1}{(t)_{\infty}} \sum_{n=0}^{\infty} \frac{(t)_{n}(-1)^{n} q^{n(t, \alpha, z ; q)}\left(q^{3} / z^{2} ; q^{2}\right)_{n}}{\left(-q^{2} / z ; q\right)_{n}} \\
\gamma(t, \alpha, z ; q)=\frac{1}{(t)_{\infty}} \sum_{n=0}^{\infty} \frac{(t)_{n} q^{n(n-3)+n \alpha} z^{2 n}}{\left(v^{2} z ; q\right)_{n}\left(v^{4} z ; q\right)_{n}} .
\end{gathered}
$$

For $t=0, \alpha=1$, we have the generalized functions of Choi.

The generalized eighth order mock theta functions:

$$
\begin{aligned}
& =\frac{1}{(t)_{\infty}} \sum_{n=0}^{\infty} \frac{(t)_{n} q^{n^{2}-2 n+n \alpha} z^{n}\left(-z^{2} / q ; q^{2}\right)_{n}}{\left(-z^{2} ; q^{2}\right)_{n}} \\
& =\frac{1}{(t)_{\infty}} \sum_{n=0}^{\infty} \frac{(t)_{n} q^{n^{2}+n \alpha} z^{n}\left(-z^{2} / q ; q^{2}\right)_{n}}{\left(-z^{2} ; q^{2}\right)_{n}}, \\
& =\frac{1}{(t)_{\infty}} \sum_{n=0}^{\infty} \frac{(t)_{n} q^{n^{2}+n+n \alpha} z^{n+2}\left(-z^{2} ; q^{2}\right)_{n}}{\left(-z^{2} / q ; q^{2}\right)_{n+1}}, \\
& =\frac{1}{(t)_{\infty}} \sum_{n=0}^{\infty} \frac{(t)_{n} q^{n^{2}-n+n \alpha} z^{n}\left(-z^{2} ; q^{2}\right)_{n}}{\left(-z^{2} / q ; q^{2}\right)_{n+1}} \\
& =\frac{1}{(t)_{\infty}} \sum_{n=0}^{\infty} \frac{(t)_{n} q^{n^{2}-3 n+n \alpha} z^{2 n}\left(-z^{2} / q ; q^{2}\right)_{n}}{\left(-z^{2} q^{2} ; q^{4}\right)_{n}} \\
& U_{1}(t, \alpha, z ; q) \\
& =\frac{1}{(t)_{\infty}} \sum_{n=0}^{\infty} \frac{(t)_{n} q^{n^{2}+n \alpha} z^{n+1}\left(-z ; q^{2}\right)_{n}}{\left(z^{2} / q ; q^{2}\right)_{n+1}} \\
& =\frac{1}{(t)_{\infty}} \sum_{n=0}^{\infty} \frac{(t)_{n} q^{n^{2}-n+n \alpha} z^{2 n+1}\left(-z^{2} / q ; q^{2}\right)_{n}}{\left(-z^{2} ; q^{4}\right)_{n+1}} \\
& V_{0}(t, \alpha, z ; q) \\
& \left(z^{2} / q ; q^{2}\right)_{n}
\end{aligned}
$$

For $t=0, \alpha=1$, and $z=q$, these generalized functions reduce to the mock theta functions of Gordon and McIntosh.

The generalized tenth order mock theta functions [6]:

$$
\begin{aligned}
& \varphi(t, \alpha, z ; q)=\frac{1}{(t)_{\infty}} \sum_{n=0}^{\infty} \frac{(t)_{n} q^{\frac{n(n-5)}{2}+n \alpha} z^{2 n}}{\left(z^{2} / q ; q^{2}\right)_{n+1}} \\
& \psi(t, \alpha, z ; q)=\frac{1}{(t)_{\infty}} \sum_{n=0}^{\infty} \frac{(t)_{n} q^{\frac{n(n-3)}{2}+n \alpha} z^{2 n+1}}{\left(z^{2} / q ; q^{2}\right)_{n+1}}
\end{aligned}
$$

$$
\begin{aligned}
& \mathrm{X}(t, \alpha, z ; q) \\
& =\frac{1}{(t)_{\infty}} \sum_{n=0}^{\infty} \frac{(-1)^{n}(t)_{n} q^{n(n-3)+n \alpha} z^{2 n}}{\left(-z^{2} / q ; q\right)_{2 n}}, \\
& =\frac{1}{(t)_{\infty}} \sum_{n=0}^{\infty} \frac{(-1)^{n}(t)_{n} q^{n(t, \alpha, z ; q)+n \alpha} z^{2 n+1}}{\left(-z^{2} / q ; q\right)_{2 n+1}} .
\end{aligned}
$$

For $t=0, \alpha=1$ and $z=q$ these generalized functions reduce to tenth order mock theta functions of Ramanujan.

\section{Generating Functions of Generalized Partial Third Order Mock Theta Functions}

(i) Taking $\alpha_{r}=\frac{q^{r^{2}-4 r+r \beta} \alpha^{r} z^{2 r}}{(-z ; q)_{r}(-\alpha z / q ; q)_{r}}$ in (1)

$$
\begin{aligned}
\sum_{m=0}^{\infty} t^{m} f_{R m} & (0, \alpha, \beta, z ; q) \\
& =\sum_{r=0}^{\infty} \frac{q^{r^{2}-4 r+r \beta} \alpha^{r} z^{2 r} t^{r}}{(-z ; q)_{r}(-\alpha z / q ; q)_{r}} \sum_{n=0}^{\infty} t^{n},
\end{aligned}
$$

or

$$
\begin{aligned}
& \sum_{m=0}^{\infty} t^{m} f_{R m}(0, \alpha, \beta, z ; q) \\
& ={ }_{1} \phi_{2}\left[\begin{array}{c}
q \\
-z,-\alpha z / q
\end{array} ; q, t \alpha z^{2} q^{\beta-3}\right] \sum_{n=0}^{\infty} t^{n} .
\end{aligned}
$$

Special case:

For $\beta=1, \alpha=q, z=q$, in (38) we get the generating function for partial mock theta function of Ramanujan

$$
\begin{gathered}
\sum_{m=0}^{\infty} t^{m} f_{R m}(q)=\sum_{r=0}^{\infty} \frac{q^{r^{2}} t^{r}}{(-q ; q)_{r}(-q ; q)_{r}} \sum_{n=0}^{\infty} t^{n} \\
={ }_{1} \phi_{2}\left[\begin{array}{c}
q \\
-q,-q ; q, t q] \sum_{n=0}^{\infty} t^{n} .
\end{array}\right.
\end{gathered}
$$

We list the generating functions for other generalized and partial mock theta functions omitting calculations only giving the value of $\alpha_{r}$ in paranthesis.

(ii) $\sum_{m=0}^{\infty} t^{m} \phi_{R m}(0, \alpha, \beta, z ; q)$
$={ }_{1} \phi_{2}\left[i z \sqrt{\alpha / q},-i z \sqrt{\alpha / q} ; q, t z^{2} q^{\beta-2}\right] \sum_{n=0}^{\infty} t^{n}$. 


$$
\left(\alpha_{r}=\frac{q^{r^{2}-3 r+r \beta} z^{2 r}}{\left(-\alpha z^{2} / q ; q^{2}\right)_{r}} \text { in (1) }\right)
$$

Here $\phi_{R m}(q)$ is the partial mock theta functions of Ramanujan.

Special cases:

$$
\begin{array}{r}
\sum_{m=0}^{\infty} t^{m} \phi_{R m}(q)={ }_{1} \phi_{2}\left[\begin{array}{c}
q \\
i q,-i q
\end{array} ; q, t q\right] \sum_{n=0}^{\infty} t^{n} . \\
(\beta=1, \alpha=q, z=q \text { in }(41))
\end{array}
$$

(ii) $\sum_{m=0}^{\infty} t^{m} \psi_{R m}(0, \alpha, \beta, z ; q)=\frac{z}{\left(1-\alpha z^{2} / q^{2}\right)}$

$$
\begin{aligned}
& \times{ }_{1} \phi_{2}\left[\begin{array}{c}
q \\
\alpha^{1 / 2} z,-\alpha^{1 / 2} z^{\prime}
\end{array} q, t z^{2} q^{\beta}\right] \sum_{n=0}^{\infty} t^{n} . \\
& \left(\alpha_{r}=\frac{q^{r^{2}-r+r \beta} z^{2 r}}{\left(\alpha z^{2} ; q^{2}\right)_{r}} \text { in (1) }\right)
\end{aligned}
$$

Here $\psi_{R m}(q)$ is the partial mock theta functions of Ramanujan.

Special cases:

$$
\begin{aligned}
& \sum_{m=0}^{\infty} t^{m} \psi_{R m}(q) \\
& =\frac{q}{(1-q)}{ }_{1} \phi_{2}\left[\begin{array}{c}
q \\
q^{3 / 2},-q^{3 / 2} ; q, t q^{3}
\end{array}\right] \sum_{n=0}^{\infty} t^{n} \text {. } \\
& (\beta=1, \alpha=q, z=q \text { in }
\end{aligned}
$$

$$
\text { (iv) } \sum_{\mathrm{m}=0}^{\infty} t^{m} v_{R m}(0, \alpha, \beta, z ; q)=
$$$$
\frac{1}{\left(1+\frac{\alpha^{2} z^{2}}{q^{3}}\right)_{1}} \phi_{2}\left[i \alpha z / \sqrt{q},-i \alpha z / \sqrt{q} ; q, t z^{2} q^{\beta-1}\right] \sum_{\mathrm{n}=0}^{\infty} t^{n} .
$$$$
\left(\alpha_{r}=\frac{q^{r^{2}-2 r+r \beta} z^{2 r}}{\left(-\alpha^{2} z^{2} / q^{3} ; q^{2}\right)_{r+1}} \text { in }(1)\right)
$$

Here $v_{R m}(q)$ is the partial mock theta functions of Ramanujan.

Special cases:

$$
\begin{gathered}
\sum_{m=0}^{\infty} t^{m} v_{R m}(q)= \\
\frac{1}{(1+q)}{ }_{1} \phi_{2}\left[\begin{array}{c}
\left.q q^{3 / 2},-i q^{3 / 2} ; q, t q^{2}\right] \sum_{n=0}^{\infty} t^{n} . \\
(\beta=1, \alpha=q, z=q \text { in })
\end{array}\right.
\end{gathered}
$$

$$
\begin{gathered}
\text { (v) } \sum_{m=0}^{\infty} t^{m} \omega_{R m}(0, \alpha, \beta, z ; q) \\
=\frac{z^{4}}{q^{4}\left(1-z^{2} / q\right)\left(1-\alpha^{2} z^{2} / q^{3}\right)} \\
\times{ }_{1} \phi_{2}\left[\begin{array}{l}
q^{2} \\
z q^{2}, \alpha^{2} z^{2} / q^{;}
\end{array} q^{2}, t \alpha^{2} z^{4} q^{\beta-3}\right] \sum_{n=0}^{\infty} t^{n} . \quad(44) \\
\left(\alpha_{r}=\frac{q^{2 r^{2}-5 r+r \beta-4} \alpha^{2 r} z^{4 r+4}}{\left(z^{2} / q ; q^{2}\right)_{r+1}\left(\alpha^{2} z^{2} / q^{3} ; q^{2}\right)_{r+1}}\right. \text { in (1)) }
\end{gathered}
$$

Here $\omega_{R m}(q)$ is the partial mock theta functions of Ramanujan.

Special cases:

$$
\begin{aligned}
& \sum_{m=0}^{\infty} t^{m} \omega_{R m}(q)=\frac{1}{(1-q)^{2}}{ }_{1} \phi_{2}\left[\begin{array}{c}
q^{2} \\
q^{3}, q^{3}
\end{array} q^{2}, t q^{4}\right] \sum_{n=0}^{\infty} t^{n} \\
& (\beta=1, \alpha=q, z=q \text { in }) \\
& \text { (vi) } \sum_{m=0}^{\infty} t^{m} \chi_{R m}(0, \beta, z ; q) \\
& ={ }_{1} \phi_{2}\left[\begin{array}{c}
q \\
v z,-v^{2} z
\end{array} ; q, t z^{2} q^{\beta-2}\right] \sum_{n=0}^{\infty} t^{n} \\
& \left(\alpha_{r}=\frac{q^{r^{2}-3 r+r \beta_{z} 2 r}}{(v z ; q)_{r}\left(-v^{2} z ; q\right)_{r}} \text { in }(1)\right)
\end{aligned}
$$

Here $\chi_{R m}(q)$ is the partial mock theta functions of Ramanujan.

Special cases:

$$
\begin{gathered}
\sum_{m=0}^{\infty} t^{m} \chi_{R m}(q)={ }_{1} \phi_{2}\left[\begin{array}{c}
q \\
v q,-v^{2} q^{;}
\end{array}, t q\right] \sum_{n=0}^{\infty} t^{n} . \\
(\beta=1, \alpha=q, z=q \text { in (45)) }
\end{gathered}
$$

$$
\text { (vii) } \begin{aligned}
\sum_{m=0}^{\infty} t^{m} \rho_{R m}(0, \beta, z ; q) & \\
& =\frac{z^{4}}{q^{4}\left(1-v^{2} z^{2} / q\right)\left(1-v^{-2} z^{2} / q\right)}
\end{aligned}
$$$$
\times{ }_{1} \phi_{2}\left[\begin{array}{c}
q^{2} \\
v^{2} z^{2} q, v^{-2} z^{2} q^{;}
\end{array} q^{2}, t z^{4} q^{\beta-1}\right] \sum_{n=0}^{\infty} t^{n} .
$$

$$
\left(\alpha_{r}=\frac{q^{2 r^{2}-3 r+r \beta} z^{4 r}}{\left(v^{2} z^{2} / q ; q^{2}\right)_{r+1}\left(v^{-2} z^{2} / q ; q^{2}\right)_{r+1}}\right. \text { in (1) }
$$

Here $\rho_{R m}(q)$ is the partial mock theta functions of Ramanujan.

Special cases:

$$
\sum_{m=0}^{\infty} t^{m} \rho_{R m}(q)=\frac{1}{\left(1-v^{2} q\right)\left(1-v^{-2} q\right)}
$$




$$
\begin{aligned}
& \times{ }_{1} \phi_{2}\left[\begin{array}{c}
q^{2} \\
v^{2} q^{3}, v^{-2} q^{3}
\end{array} q^{2}, t q^{4}\right] \sum_{n=0}^{\infty} t^{n} \\
& (\beta=1, \alpha=q, z=q \text { in }(46))
\end{aligned}
$$

\section{Generating Functions of Generalized} Partial Fifth Order Mock Theta

\section{Functions}

$$
\begin{aligned}
& \text { (i) } \sum_{m=0}^{\infty} t^{m} f_{0 m}(0, \alpha, z ; q) \\
& ={ }_{1} \phi_{2}\left[\begin{array}{c}
q \\
-z, 0
\end{array} ;, t z^{2} q^{\alpha-2}\right] \sum_{n=0}^{\infty} t^{n} \text {. } \\
& \left(\alpha_{r}=\frac{q^{r^{2}-3 r+r \alpha} z^{2 r}}{(-z ; q) r}\right. \text { in (1)) }
\end{aligned}
$$

Here $f_{0 m}(q)$ is the partial mock theta functions of Ramanujan.

Special cases:

$$
\begin{gathered}
\sum_{m=0}^{\infty} t^{m} f_{0 m}(q)={ }_{1} \phi_{2}\left[\begin{array}{c}
q \\
-q, 0 ;
\end{array} ;, t q\right] \sum_{n=0}^{\infty} t^{n} . \\
(\beta=1, \alpha=q, z=q \text { in }(47)) \\
\text { (ii) } \sum_{m=0}^{\infty} t^{m} \phi_{0 m}(0, \alpha, z ; q) \\
={ }_{2} \phi_{2}\left[\begin{array}{c}
q^{2},-q^{3} / z^{2} \\
0,0
\end{array} q^{2},-t z^{-2} q^{\alpha+2}\right] \sum_{n=0}^{\infty} t^{n} . \\
\left(\alpha_{r}=\frac{q^{r^{2}+r+r \alpha}\left(-q^{3} / z^{2} ; q^{2}\right)_{r}}{z^{2 r}} \text { in }(1)\right)
\end{gathered}
$$

Here $\phi_{0 m}(q)$ is the partial mock theta functions of Ramanujan.

Special cases:

$$
\begin{aligned}
& \sum_{m=0}^{\infty} t^{m} \phi_{0 m}(q)={ }_{2} \phi_{2}\left[\begin{array}{c}
q^{2},-q \\
0,0
\end{array} ; q^{2},-t q\right] \sum_{n=0}^{\infty} t^{n} . \\
& (\beta=1, \alpha=q, z=q \text { in (48)) } \\
& \text { (iii) } \sum_{m=0}^{\infty} t^{m} \psi_{0 m}(0, \alpha, z ; q) \\
& =\frac{q^{\alpha+1}}{z}{ }_{2} \phi_{2}\left[\begin{array}{c}
q,-q^{2} / z \\
0,0
\end{array} q,-t z^{-1} q^{\alpha+2}\right] \sum_{n=0}^{\infty} t^{n} . \\
& \left(\alpha_{r}=\frac{q^{r^{2}+3 r / 2+r \alpha}\left(-q^{2} / z ; q\right)_{r}}{z^{r}} \text { in }(1)\right)
\end{aligned}
$$

Here $\psi_{0 m}(q)$ is the partial mock theta functions of Ramanujan.
Special cases:

$$
\begin{array}{r}
\sum_{m=0}^{\infty} t^{m} \psi_{0 m}(q)=q_{2} \phi_{2}\left[\begin{array}{c}
q,-q \\
0,0
\end{array} ; q,-t q^{2}\right] \sum_{n=0}^{\infty} t^{n} . \\
(\beta=1, \alpha=q, z=q \text { in (49)) }
\end{array}
$$

$$
\begin{aligned}
& \text { (iv) } \sum_{m=0}^{\infty} t^{m} F_{0 m}(0, \alpha, z ; q) \\
& ={ }_{1} \phi_{2}\left[\begin{array}{c}
q^{2} \\
z^{2} / q, 0
\end{array} ; q^{2}, t z^{4} q^{\alpha-3}\right] \sum_{n=0}^{\infty} t^{n} . \\
& \left(\alpha_{r}=\frac{q^{2 r^{2}-5 r+r \alpha_{z} 4 r}}{\left(z^{2} / q ; q^{2}\right)_{r}} \text { in (1) }\right)
\end{aligned}
$$

Here $F_{0 m}(q)$ is the partial mock theta functions of Ramanujan.

Special cases:

$$
\begin{aligned}
& \sum_{m=0}^{\infty} t^{m} F_{0 m}(q)={ }_{1} \phi_{2}\left[\begin{array}{c}
q^{2} \\
q, 0^{2}
\end{array} q^{2}, t q^{2}\right] \sum_{n=0}^{\infty} t^{n} . \\
& (\beta=1, \alpha=q, z=q \text { in (50)) } \\
& \text { (v) } \sum_{m=0}^{\infty} t^{m} f_{1 m}(0, \alpha, z ; q) \\
& ={ }_{1} \phi_{2}\left[\begin{array}{c}
q \\
-z, 0
\end{array} ; q, t z^{2} q^{\alpha-1}\right] \sum_{n=0}^{\infty} t^{n} . \\
& \left(\alpha_{r}=\frac{q^{r^{2}-2 r+r \alpha_{z} 2 r}}{(-z ; q)_{r}}\right. \text { in (1)) }
\end{aligned}
$$

Here $f_{1 m}(q)$ is the partial mock theta functions of Ramanujan.

Special cases:

$$
\begin{aligned}
& \sum_{m=0}^{\infty} t^{m} f_{1 m}(q)={ }_{1} \phi_{2}\left[\begin{array}{c}
q \\
-q, 0
\end{array} ; q, t q^{2}\right] \sum_{n=0}^{\infty} t^{n} . \\
& (\beta=1, \alpha=q, z=q \text { in (51) }) \\
& \text { (vi) } \sum_{m=0}^{\infty} t^{m} \phi_{1 m}(0, \alpha, z ; q) \\
& =\frac{q^{5}}{z^{4}}{ }_{2} \phi_{2}\left[\begin{array}{c}
q^{2},-q^{3} / z^{2} \\
0,0
\end{array} ; q^{2},-t z^{-2} q^{\alpha+4}\right] \sum_{n=0}^{\infty} t^{n} \text {. } \\
& \left(\alpha_{r}=\frac{q^{r^{2}+3 r+r \alpha}\left(-q^{3} / z^{2} ; q^{2}\right)_{r}}{z^{2 r}} \text { in (1) }\right)
\end{aligned}
$$

Here $\phi_{1 m}(q)$ is the partial mock theta functions of Ramanujan.

Special cases:

$$
\sum_{m=0}^{\infty} t^{m} \phi_{1 m}(q)=q_{2} \phi_{2}\left[\begin{array}{c}
q^{2},-q \\
0,0
\end{array} ; q^{2},-t q^{3}\right] \sum_{n=0}^{\infty} t^{n} \text {. }
$$




$$
(\beta=1, \alpha=q, z=q \text { in (52) }
$$

(vii) $\sum_{m=0}^{\infty} t^{m} \psi_{1 m}(0, \alpha, z ; q)$

$=\frac{q}{z}{ }_{2} \phi_{2}\left[\begin{array}{c}q,-q^{2} / z \\ 0,0\end{array} ;,-t z^{-1} q^{\alpha+1}\right] \sum_{n=0}^{\infty} t^{n}$.

$$
\left(\alpha_{r}=\frac{q^{\left(r^{2}+r\right) / 2+r \alpha}\left(-q^{2} / z ; q\right)_{r}}{z^{r}} \text { in (1) }\right)
$$

Here $\psi_{1 m}(q)$ is the partial mock theta functions of Ramanujan.

Special cases:

$$
\begin{aligned}
& \sum_{m=0}^{\infty} t^{m} \psi_{1 m}(q)={ }_{2} \phi_{2}\left[\begin{array}{c}
q,-q \\
0,0
\end{array} ; q,-t q\right] \sum_{n=0}^{\infty} t^{n} . \\
& (\beta=1, \alpha=q, z=q \text { in (53)) } \\
& \text { (vii) } \sum_{m=0}^{\infty} t^{m} F_{1 m}(0, \alpha, z ; q) \\
& =\frac{1}{\left(1-z^{2} / q\right)}{ }_{1} \phi_{2}\left[\begin{array}{c}
q^{2} \\
z^{2} q, 0^{\prime}
\end{array} q^{2}, t z^{4} q^{\alpha-1}\right] \sum_{n=0}^{\infty} t^{n} . \\
& \left(\alpha_{r}=\frac{q^{2 r^{2}-3 r+r \alpha} z^{4 r}}{\left(z^{2} / q ; q^{2}\right) r+1} \text { in }(1)\right)
\end{aligned}
$$

Here $F_{1 m}(q)$ is the partial mock theta functions of Ramanujan.

Special cases:

$$
\begin{gathered}
\sum_{m=0}^{\infty} t^{m} F_{1 m}(q)=\frac{1}{(1-q)}{ }_{1} \phi_{2}\left[\begin{array}{c}
q^{2} \\
q^{3}, 0^{3}
\end{array} q^{2}, t q^{4}\right] \sum_{n=0}^{\infty} t^{n} . \\
(\beta=1, \alpha=q, z=q \text { in }(54))
\end{gathered}
$$

\section{Generating Functions of Generalized Partial Sixth Order Mock Theta \\ Functions}

$$
\begin{aligned}
& \text { (i) } \sum_{m=0}^{\infty} t^{m} \Phi_{m}(0, \alpha, z ; q) \\
& ={ }_{2} \phi_{2}\left[\begin{array}{c}
q^{2}, z^{2} / q \\
-z^{2} / q,-z^{2} ; q^{2}, t z^{2} q^{\alpha-2}
\end{array}\right] \sum_{n=0}^{\infty} t^{n} . \\
& \quad\left(\alpha_{r}=\frac{(-1)^{r} q^{r^{2}-3 r+r \alpha_{z^{2 r}}\left(z^{2} / q ; q^{2}\right)_{r}}}{\left.\left(-z^{2} / q ; q\right)\right)_{2 r}} \text { in }(1)\right)
\end{aligned}
$$

Here $\Phi_{m}(q)$ is the partial mock theta functions of Ramanujan.

Special cases:

$\sum_{m=0}^{\infty} t^{m} \Phi_{m}(q)={ }_{2} \phi_{2}\left[\begin{array}{c}q^{2}, q \\ -q,-q^{2}\end{array} ; q^{2}, t q\right] \sum_{n=0}^{\infty} t^{n}$.

$$
(\beta=1, \alpha=q, z=q \text { in (55)) }
$$

(ii) $\sum_{m=0}^{\infty} t^{m} \Psi_{m}(0, \alpha, z ; q)$

$=\frac{z}{\left(1+z^{2} / q\right)}{ }_{2} \phi_{2}\left[\begin{array}{c}q^{2}, z^{2} / q \\ -z^{2},-z^{2} q^{2}\end{array} q^{2}, t z^{2} q^{\alpha}\right] \sum_{n=0}^{\infty} t^{n}$.

$$
\left(\alpha_{r}=\frac{(-1)^{r} q^{r^{2}-r+r \alpha^{2}} z^{2 r+1}\left(z^{2} / q ; q^{2}\right)_{r}}{\left(-z^{2} / q ; q\right)_{2 r+1}}\right. \text { in (1) }
$$

Here $\Psi_{m}(q)$ is the partial mock theta functions of Ramanujan.

Special cases:

$$
\begin{gathered}
\sum_{m=0}^{\infty} t^{m} \Psi_{m}(q)=\frac{q}{(1+q)}{ }_{2} \phi_{2}\left[\begin{array}{c}
q^{2}, q \\
-q^{2},-q^{3}
\end{array} ; q^{2}, t q^{3}\right] \sum_{n=0}^{\infty} t^{n} . \\
(\beta=1, \alpha=q, z=q \text { in }(56)) \\
\text { (iii) } \sum_{m=0}^{\infty} t^{m} \rho_{m}(0, \alpha, z ; q)=\frac{1}{\left(1-z^{2} / q\right)} \\
\times_{2} \phi_{2}\left[\begin{array}{c}
q,-z \\
\left.z \sqrt{q},-z \sqrt{q} ; q,-t z q^{\alpha-1}\right]
\end{array} \sum_{n=0}^{\infty} t^{n} .(57)\right. \\
\left(\alpha_{r}=\frac{q^{r(r-3) / 2+r \alpha_{z} r(-z ; q)} r}{\left(z^{2} / q ; q^{2}\right)_{r+1}} \text { in }(1)\right)
\end{gathered}
$$

Here $\rho_{m}(q)$ is the partial mock theta functions of Ramanujan.

Special cases:

$$
\begin{aligned}
& \sum_{m=0}^{\infty} t^{m} \rho_{m}(q) \\
& =\frac{1}{(1-q)}{ }_{2} \phi_{2}\left[\begin{array}{c}
q,-q \\
q^{3 / 2},-q^{3 / 2} ; q,-t q
\end{array}\right] \sum_{n=0}^{\infty} t^{n} . \\
& (\beta=1, \alpha=q, z=q \text { in (57) } \\
& \text { (iv) } \sum_{m=0}^{\infty} t^{m} \gamma_{m}(0, \alpha, z ; q) \\
& ={ }_{1} \phi_{2}\left[\begin{array}{c}
q, \\
v^{2} z, v^{4} z^{\prime}
\end{array} q, t z^{2} q^{\alpha-2}\right] \sum_{n=0}^{\infty} t^{n} . \\
& \left(\alpha_{r}=\frac{q^{r^{2}-3 r+r \alpha} z^{2 r}}{\left(v^{2} z ; q\right)_{r}\left(v^{4} z ; q\right)_{r}} \text { in (1) }\right)
\end{aligned}
$$

Here $\gamma_{m}(q)$ is the partial mock theta functions of Ramanujan.

Special cases:

$\sum_{m=0}^{\infty} t^{m} \gamma_{m}(q)={ }_{1} \phi_{2}\left[\begin{array}{c}q, \\ v^{2} q, v^{4} q^{;}\end{array} q, t q\right] \sum_{n=0}^{\infty} t^{n}$. 


$$
\begin{aligned}
& (\beta=1, \alpha=q, z=q \text { in (58)) } \\
& \text { (v) } \sum_{m=0}^{\infty} t^{m} \sigma_{m}(0, \alpha, z ; q) \\
& =\frac{z(1+z / q)}{2\left(1-z^{2} / q\right)}{ }_{2} \phi_{2}\left[\begin{array}{c}
q,-z \\
z \sqrt{q},-z \sqrt{q} ; q,-t z q^{\alpha}
\end{array}\right] \sum_{n=0}^{\infty} t^{n} \text {. } \\
& \left(\alpha_{r}=\frac{q^{\left(r^{2}-r\right) / 2+r \alpha} z^{r+1}(-z / q ; q)_{r+1}}{2\left(z^{2} / q ; q^{2}\right)_{r+1}} \text { in (1) }\right)
\end{aligned}
$$

Here $\sigma_{m}(q)$ is the partial mock theta functions of Ramanujan.

Special cases:

$$
\begin{aligned}
& \sum_{m=0}^{\infty} t^{m} \sigma_{m}(q) \\
& =\frac{q}{(1-q)}{ }_{2} \phi_{2}\left[\begin{array}{c}
q,-q \\
q^{3 / 2},-q^{3 / 2} ; q,-t q^{2}
\end{array}\right] \sum_{n=0}^{\infty} t^{n} \\
& (\beta=1, \alpha=q, z=q \text { in }(59))
\end{aligned}
$$

\section{Generating Functions of Generalized} Partial Eighth Order Mock Theta

\section{Functions}

$$
\begin{aligned}
\text { (i) } \sum_{m=0}^{\infty} t^{m} \mathrm{~S}_{0 m}(0, \alpha, z ; q) \\
={ }_{2} \phi_{2}\left[\begin{array}{c}
q^{2},-z^{2} / q \\
-z^{2}, 0
\end{array} ; q^{2},-t z q^{\alpha-1}\right] \sum_{n=0}^{\infty} t^{n} . \\
\left(\alpha_{r}=\frac{q^{r^{2}-2 r+r \alpha} z^{r}\left(-z^{2} / q ; q^{2}\right)_{r}}{\left(-z^{2} ; q^{2}\right)_{r}} \text { in }(1)\right)
\end{aligned}
$$

Here $\mathrm{S}_{0 m}(q)$ is the partial mock theta functions of Gordon and McIntosh.

Special cases:

$$
\begin{gathered}
\sum_{m=0}^{\infty} t^{m} \mathrm{~S}_{0 m}(q)={ }_{2} \phi_{2}\left[\begin{array}{l}
q^{2},-q \\
-q^{2}, 0
\end{array} q^{2},-t q\right] \sum_{n=0}^{\infty} t^{n} . \\
(\beta=1, \alpha=q, z=q \text { in }(60)) \\
\text { (ii) } \sum_{m=0}^{\infty} t^{m} \mathrm{~S}_{1 m}(0, \alpha, z ; q) \\
={ }_{2} \phi_{2}\left[\begin{array}{c}
q^{2},-z^{2} / q \\
-z^{2}, 0
\end{array} q^{2},-t z q^{\alpha+1}\right] \sum_{n=0}^{\infty} t^{n} . \\
\left(\alpha_{r}=\frac{q^{r^{2}+r \alpha_{z} r}\left(-z^{2} / q ; q^{2}\right)_{r}}{\left(-z^{2} ; q^{2}\right)_{r}} \text { in }(1)\right)
\end{gathered}
$$

Here $S_{1 m}(q)$ is the partial mock theta functions of Gordon and McIntosh.

Special cases:

$$
\begin{aligned}
& \sum_{m=0}^{\infty} t^{m} \mathrm{~S}_{1 m}(q)={ }_{2} \phi_{2}\left[\begin{array}{l}
q^{2},-q \\
-q^{2}, 0
\end{array} q^{2},-t q^{3}\right] \sum_{n=0}^{\infty} t^{n} \\
& (\beta=1, \alpha=q, z=q \text { in }(61)) \\
& \text { (iii) } \sum_{m=0}^{\infty} t^{m} \mathrm{~T}_{0 m}(0, \alpha, z ; q) \\
& =\frac{z^{2}}{\left(1+z^{2} / q\right)}{ }_{2} \phi_{2}\left[\begin{array}{l}
q^{2},-z^{2} \\
-z^{2} q, 0^{2}
\end{array} q^{2},-t z q^{\alpha+2}\right] \sum_{n=0}^{\infty} t^{n} . \\
& \left(\alpha_{r}=\frac{q^{r^{2}+r+r \alpha} z^{r+2}\left(-z^{2} ; q^{2}\right)_{r}}{\left(-z^{2} / q ; q^{2}\right)_{r+1}} \text { in }(1)\right)
\end{aligned}
$$

Here $\mathrm{T}_{0 m}(q)$ is the partial mock theta functions of Gordon and McIntosh.

Special cases:

$$
\begin{gathered}
\sum_{m=0}^{\infty} t^{m} \mathrm{~T}_{0 m}(q)=\frac{q^{2}}{(1+q)}{ }_{2} \phi_{2}\left[\begin{array}{c}
q^{2},-q^{2} \\
-q^{3}, 0
\end{array} ; q^{2},-t q^{4}\right] \sum_{n=0}^{\infty} t^{n} . \\
(\beta=1, \alpha=q, z=q \text { in (62) })
\end{gathered}
$$

(iv) $\sum_{m=0}^{\infty} t^{m} \mathrm{~T}_{1 m}(0, \alpha, z ; q)$

$$
\begin{gathered}
=\frac{1}{\left(1+z^{2} / q\right)}{ }_{2} \phi_{2}\left[\begin{array}{l}
q^{2},-z^{2} \\
-z^{2} q, 0^{2} ; q^{2},-t z q^{\alpha}
\end{array}\right] \sum_{n=0}^{\infty} t^{n} . \\
\left(\alpha_{r}=\frac{q^{r^{2}-r+r \alpha_{z} r}\left(-z^{2} ; q^{2}\right)_{r}}{\left(-z^{2} / q ; q^{2}\right)_{r+1}} \text { in }(1)\right)
\end{gathered}
$$

Here $\mathrm{T}_{1 m}(q)$ is the partial mock theta functions of Gordon and McIntosh.

Special cases:

$$
\begin{gathered}
\sum_{m=0}^{\infty} t^{m} \mathrm{~T}_{1 m}(q)=\frac{1}{(1+q)}{ }_{2} \phi_{2}\left[\begin{array}{c}
q^{2},-q^{2} \\
-q^{3}, 0
\end{array} ; q^{2},-t q^{2}\right] \sum_{n=0}^{\infty} t^{n} \\
(\beta=1, \alpha=q, z=q \text { in }(63)) \\
\text { (v) } \sum_{m=0}^{\infty} t^{m} \mathrm{U}_{0 m}(0, \alpha, z ; q) \\
={ }_{2} \phi_{2}\left[\begin{array}{c}
q^{2},-z^{2} / q \\
i z q,-i z q
\end{array} q^{2},-t z^{2} q^{\alpha-2}\right] \sum_{n=0}^{\infty} t^{n} . \quad(64) \\
\left(\alpha_{r}=\frac{q^{r^{2}-3 r+r z^{2 r}\left(-z^{2} / q ; q^{2}\right)_{r}}}{\left(-z^{2} q^{2} ; q^{4}\right)_{r}} \text { in (1) }\right)
\end{gathered}
$$

Here $\mathrm{U}_{0 m}(q)$ is the partial mock theta functions of Gordon and McIntosh.

Special cases:

$$
\sum_{m=0}^{\infty} t^{m} \mathrm{U}_{0 m}(q)={ }_{2} \phi_{2}\left[\begin{array}{c}
q^{2},-q \\
i q^{2},-i q^{2}
\end{array} ; q^{2},-t q\right] \sum_{n=0}^{\infty} t^{n} .
$$




$$
\begin{aligned}
& \qquad \beta=1, \alpha=q, z=q \text { in }(64)) \\
& \text { (vi) } \sum_{m=0}^{\infty} t^{m} \mathrm{U}_{1 m}(0, \alpha, z ; q) \\
& =\frac{z}{\left(1+z^{2}\right)}{ }_{2} \phi_{2}\left[\begin{array}{c}
q^{2},-z^{2} / q \\
i z q^{2},-i z q^{2}
\end{array} q^{2},-t z^{2} q^{\alpha}\right] \sum_{n=0}^{\infty} t^{n} \\
& \left(\alpha_{r}=\frac{q^{r^{2}-r+r \alpha} z^{2 r+1}\left(-z^{2} / q ; q^{2}\right)_{r}}{\left(-z^{2} ; q^{4}\right)_{r+1}} \text { in }(1)\right)
\end{aligned}
$$

Here $\mathrm{U}_{1 m}(q)$ is the partial mock theta functions of Gordon and McIntosh.

$$
\text { Special cases: }
$$

$$
\begin{aligned}
& \sum_{m=0}^{\infty} t^{m} \mathrm{U}_{1 m}(q) \\
& =\frac{q}{\left(1+q^{2}\right)}{ }_{2} \phi_{2}\left[\begin{array}{c}
q^{2},-q \\
i q^{3},-i q^{3}
\end{array} ; q^{2},-t q^{3}\right] \sum_{n=0}^{\infty} t^{n} . \\
& (\beta=1, \alpha=q, z=q \text { in (65)) } \\
& \text { (vii) } \sum_{m=0}^{\infty} t^{m} \mathrm{~V}_{0 m}(0, \alpha, z ; q) \\
& =-1+2{ }_{2} \phi_{2}\left[\begin{array}{c}
q^{2},-z \\
z^{2} / q, 0
\end{array} ; q^{2},-t z q^{\alpha-1}\right] \sum_{n=0}^{\infty} t^{n} \text {. } \\
& \left(\alpha_{r}=\frac{q^{r^{2}-2 r+r \alpha} z^{r}\left(-z ; q^{2}\right)_{r}}{\left(z^{2} / q ; q^{2}\right)_{r}}\right. \text { in (1)) }
\end{aligned}
$$

Here $\mathrm{V}_{0 m}(q)$ is the partial mock theta functions of Gordon and McIntosh.

Special cases:

$$
\begin{aligned}
& \sum_{m=0}^{\infty} t^{m} \mathrm{~V}_{0 m}(q) \\
& =-1+2{ }_{2} \phi_{2}\left[\begin{array}{c}
q^{2},-q \\
q, 0
\end{array} q^{2},-t q\right] \sum_{n=0}^{\infty} t^{n} . \\
& (\beta=1, \alpha=q, z=q \text { in }(66)) \\
& (\text { viii }) \sum_{m=0}^{\infty} t^{m} \mathrm{~V}_{1 m}(0, \alpha, z ; q) \\
& =\frac{z}{\left(1-z^{2} / q\right)}{ }_{2} \phi_{2}\left[\begin{array}{l}
q^{2},-z \\
z^{2} q, 0
\end{array} q^{2},-t z q^{\alpha+1}\right] \sum_{n=0}^{\infty} t^{n} . \\
& \left(\alpha, \alpha_{r}=\frac{q^{r^{2}+r \alpha} z^{r+1}\left(-z ; q^{2}\right)_{r}}{\left(z^{2} / q ; q^{2}\right)_{r+1}} \text { in }(1)\right)
\end{aligned}
$$

Here $V_{1 m}(q)$ is the partial mock theta functions of Gordon and McIntosh.

Special cases:

$$
\begin{gathered}
\sum_{m=0}^{\infty} t^{m} \mathrm{~V}_{1 m}(q)=\frac{q}{(1-q)}{ }_{2} \phi_{2}\left[\begin{array}{c}
q^{2},-q \\
q^{3}, 0
\end{array} ; q^{2},-t q^{3}\right] \sum_{n=0}^{\infty} t^{n} . \\
(\beta=1, \alpha=q, z=q \text { in }(67))
\end{gathered}
$$

\section{Generating Functions of Generalized} Partial Tenth Order Mock Theta Functions

$$
\begin{aligned}
& \text { (i) } \sum_{m=0}^{\infty} t^{m} \varphi_{m}(0, \alpha, z ; q) \\
& =\frac{1}{\left(1-z^{2} / q\right)}{ }_{2} \phi_{2}\left[\begin{array}{c}
q, 0 \\
z q^{1 / 2},-z q^{1 / 2}
\end{array} ;,-t z^{2} q^{\alpha-2}\right] \sum_{n=0}^{\infty} t^{n} . \\
& \left(\alpha_{r}=\frac{q^{\left(r^{2}-5 r\right) / 2+r \alpha} z^{2 r}}{\left(z^{2} / q ; q^{2}\right) r+1}\right. \text { in (1)) }
\end{aligned}
$$

Here $\varphi_{m}(q)$ is the partial mock theta functions of Ramanujan.

Special cases:

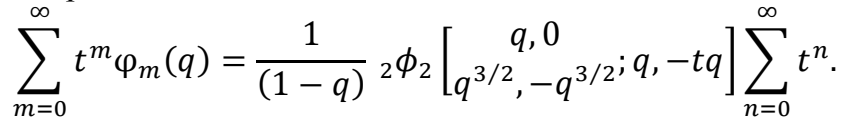

$$
\begin{aligned}
& (\beta=1, \alpha=q, z=q \text { in (68) }) \\
& \text { (ii) } \sum_{m=0}^{\infty} t^{m} \psi_{m}(0, \alpha, z ; q) \\
& =\frac{z}{\left(1-z^{2} / q\right)}{ }_{2} \phi_{2}\left[\begin{array}{c}
q, 0 \\
z q^{1 / 2},-z q^{1 / 2}
\end{array} ;,-t z^{2} q^{\alpha-1}\right] \sum_{n=0}^{\infty} t^{n} . \\
& \left(\alpha_{r}=\frac{q^{r(r-3) / 2+r \alpha} z^{2 r+1}}{\left(z^{2} / q ; q^{2}\right)_{r+1}}\right. \text { in }
\end{aligned}
$$

Here $\psi_{m}(q)$ is the partial mock theta functions of Ramanujan.

$$
\begin{aligned}
& \text { Special cases: } \\
& \sum_{m=0}^{\infty} t^{m} \psi_{m}(q) \\
& =\frac{q}{(1-q)}{ }_{2} \phi_{2}\left[\begin{array}{c}
q, 0 \\
\left.q^{3 / 2},-q^{3 / 2} ; q,-t q^{2}\right] \sum_{n=0}^{\infty} t^{n}
\end{array}\right. \\
& (\beta=1, \alpha=q, z=q \text { in }(69)) \\
& \text { (iii) } \sum_{m=0}^{\infty} t^{m} \mathrm{X}_{m}(0, \alpha, z ; q) \\
& ={ }_{2} \phi_{2}\left[-z^{2} / q,-z^{2} ; q^{2}, t z^{2} q^{\alpha-2}\right] \sum_{n=0}^{\infty} t^{n} \\
& \quad\left(\alpha_{r}=\frac{(-1)^{r} q^{r^{2}-3 r+r{ }_{z} 2 r}}{\left(-z^{2} / q ; q\right)_{2 r}} \text { in }(1)\right)
\end{aligned}
$$


Here $\mathrm{X}_{m}(q)$ is the partial mock theta functions of Ramanujan.

Special cases:

$$
\begin{gathered}
\sum_{m=0}^{\infty} t^{m} \mathrm{X}_{m}(q)={ }_{2} \phi_{2}\left[\begin{array}{c}
q^{2}, 0 \\
-q,-q^{2}
\end{array} ; q^{2}, t q\right] \sum_{n=0}^{\infty} t^{n} . \\
(\beta=1, \alpha=q, z=q \text { in }(70))
\end{gathered}
$$

(iv) $\sum_{m=0}^{\infty} t^{m} \chi_{m}(0, \alpha, z ; q)$

$=\frac{z}{\left(1+z^{2} / q\right)}{ }_{2} \phi_{2}\left[\begin{array}{c}q^{2}, 0 \\ -z^{2},-z^{2} q^{\prime}\end{array} ; q^{2}, t z^{2} q^{\alpha}\right] \sum_{n=0}^{\infty} t^{n}$.

$$
\left(\alpha_{r}=\frac{(-1)^{r} q^{r^{2}-r+r \alpha_{z} 2 r+1}}{\left(-z^{2} / q ; q\right)_{2 r+1}} \text { in }(1)\right)
$$

Here $\chi_{m}(q)$ is the partial mock theta functions of Ramanujan.

$$
\begin{aligned}
& \text { Special cases: } \\
& \sum_{m=0}^{\infty} t^{m} \chi_{m}(q) \\
& =\frac{q}{(1+q)}{ }_{2} \phi_{2}\left[\begin{array}{c}
q^{2}, 0 \\
-q^{2},-q^{3}
\end{array} ; q^{2}, t q^{3}\right] \sum_{n=0}^{\infty} t^{n} . \\
& \quad(\beta=1, \alpha=q, z=q \text { in (71)) }
\end{aligned}
$$

\section{Acknowledgement}

I am thankful to Dr. Bhaskar Srivastava for his help and guidance.

\section{References}

[1] R. P. Agarwal, Certain basic hypergeometric identities associated with mock theta functions, Quart. J. Math. (Oxford) 20 (1968), 121-128.

[2] G. E. Andrews, On basic hypergeometric series, mock theta functions and partitions (I), Quart. J. Math. (Oxford) (2) 17 (1966), 64-80.

[3] R. P. Agarwal, Certain basic hypergeometric identities associated with mock theta functions, Quart. J. Math. (Oxford) 20 (1968), 121-128.

[4] G. E. Andrews, On basic hypergeometric series, mock theta functions and partitions (I), Quart. J. Math. (Oxford) (2) 17 (1966), 64-80.

[5] S. Ramanujan, Collected Papers, Cambridge University Press, 1972, reprinted Chelsea, New York, 1962.

[6] T. M. Rassias, S.N. Singh and H.M. Srivastava, Some qgenerating functions associated with basic multiple hypergeometric series, Comp. and Math. with app. 27(1) (1994), 33-39.

[7] A. K. Srivastava, On partial sum of mock theta functions of order three, Proc. India Acad. Sci. 107 (1997), 1-12.

[8] B. Srivastava, Ramanujan's fifth order and tenth order mock theta functions- A generalization (Communicated).

[9] B. Srivastava, On a generalization of Ramanujan's seventh order mock theta functions (Accepted).

[10] B. Srivastava, Ramanujan's mock theta functions, Math. J. Okayama Univ. 47 (2005), 163-174.

[11] S. Saba, A study of a generalization of Ramanujan's sixth order and third order mock theta functions, Appl. Math. 2(5), (2012), 157-165.

[12] G.N. Watson, The final problem: An account of the mock theta functions, J. London Math. Soc. 11 (1936) 55-80. 